\title{
Same-sign dileptons as a signature for heavy Majorana neutrinos in hadron-hadron collisions
}

\author{
F. M. L. Almeida Jr. ${ }^{(1) *}$ Y. A. Coutinho ${ }^{(1) \dagger}$, J. A. Martins $\operatorname{Simões}^{(1)} \ddagger$ \\ P. P. Queiroz Filho ${ }^{(2)}$ and C. M. Porto ${ }^{(3)}$ \\ ${ }^{(1)}$ Instituto de Física \\ Universidade Federal do Rio de Janeiro, \\ (2) Departamento de Física Nuclear e Altas Energias \\ Universidade Estadual do Rio de Janeiro, \\ Rio de Janeiro \\ (3) Instituto de Ciências Exatas \\ Universidade Federal Rural do Rio de Janeiro \\ Seropédica \\ RJ, Brazil
}

\begin{abstract}
We discuss the possibility of same-sign dileptons as a signature for Majorana neutrinos. The production mechanism is given by a single heavy neutrino production and decay $p p \longrightarrow l^{ \pm} N X \longrightarrow l^{ \pm} l^{ \pm} X^{\prime}$. Cross section and distributions are presented for the LHC energies.
\end{abstract}

PACS: 12.60.-i, 13.85.-t, 14.60.St

\footnotetext{
*marroqui@if.ufrj.br

†yara@if.ufrj.br

${ }_{\ddagger}^{\ddagger}$ simoes@.if.ufrj.br

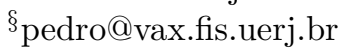


In many extensions of the standard model such as left-right models, $\mathrm{SO}(10)$ and $E_{6}$ models we have the possibility of new heavy neutrinos [1]. Heavy neutrinos are expected to play a central role in the understanding of the mechanism responsible for small masses for standard neutrinos. In the near future, direct neutrino detection with masses of a few $\mathrm{TeV}$ are feasible only at CERN high energy proton-proton collider LHC.

In this paper we turn our attention to the possibility of the production of a single heavy Majorana neutrino at the LHC energies. The case of pair production is also possible and it was studied same time ago 2]. There is a recent experimental search for pair production of heavy neutrinos at LEP 1.5 with negative results[3]. We would like to call attention that in most cases it was considered the possibility of a fourth generation heavy neutrino with standard couplings to the $Z^{0}$. But this is not the only possibility. If we also have mixing betweeen light and heavy states in the same family, then the $N N Z$ vertex is suppressed by a factor $\sin ^{2} \theta_{m i x}$. On the other hand, vertices of the type $\bar{\nu} N Z, e N W$ are suppressed only by $\sin \theta_{\text {mix }}$. We have then the more interesting possibility of single heavy neutrino production.

In see-saw models the light-to-heavy mixing is too small to give detectable effects but there are other possibilities. We can have models with new heavy neutrinos with a mixing angle which is independent of mass ratios. This is the case for models with more than one isosinglet right-handed neutrino [4 or in unified theories with B-L breaking [5]. In order to fix notation we add right-handed neutrino components to the standard families,

$$
\left(\begin{array}{c}
\nu_{e}^{0} \\
e
\end{array}\right)_{L}, \nu_{e R}^{0}, e_{R} ; \quad\left(\begin{array}{c}
\nu_{\mu}^{0} \\
\mu
\end{array}\right)_{L}, \nu_{\mu R}^{0}, \mu_{R} ; \ldots
$$

For the eigenstates,

$$
\begin{aligned}
& \chi_{\nu i}=\nu_{i L}^{0}+\left(\nu_{i L}^{0}\right)^{c} \\
& \omega_{\nu i}=\nu_{i R}^{0}+\left(\nu_{i R}^{0}\right)^{c}
\end{aligned}
$$

the mass matrix has the form:

$$
\left(\begin{array}{cc}
0 & D \\
D^{T} & M_{R}
\end{array}\right)
$$

One can diagonalize this matrix by a unitary matrix $U$, such that $\tilde{M}=$ $U^{T} M U=$ (diagonal, real). The mass eigenstates are written as combinations 
of interacting states:

$$
\begin{aligned}
\chi_{\nu i}=\sum_{k=1}^{2 n} U_{i k} \rho_{k}, & i=1,2, \ldots, n \\
\omega_{\nu i}=\sum_{k=1}^{2 n} U_{i k} \rho_{k}, & i=n+1, \ldots, 2 n
\end{aligned}
$$

where " $\mathrm{n}$ " is the family index. The mass matrix can be diagonalized by blocks [6] through the matrix product:

$$
U=U^{\prime} V=\left[\begin{array}{cc}
\left(1-\frac{1}{2} \xi^{*} \xi^{T}\right) V_{1} & \xi^{*} V_{2} \\
-\xi^{T} V_{1} & \left(1-\frac{1}{2} \xi \xi^{*}\right) V_{2}
\end{array}\right]+O\left(\xi^{3}\right)
$$

where $\xi=D M_{R}^{-1}$ and $V_{1}, V_{2}$ are unitary matrices. In the see-saw mechanism $M_{R}$ is very large and the standard neutrinos are very light. In this case the mixing parameters become very small. An alternative point of view is to have some singular mass matrix [4], [7]. This implies in a nearly zero eigenvalue for the known neutrinos masses and the mixing angles are free parameters, fixed from phenomenological constraints. Then the mixing parameters for light to heavy leptonic transitions can be large even if the neutrino mass spectrum contains very light and heavy states.

The lagrangians relevants for our purpose are:

$$
\begin{aligned}
\mathcal{L}_{c c} & =-\frac{g}{2 \sqrt{2}} W_{\mu} \sum_{i=1}^{n} \bar{l}_{i} \gamma^{\mu}\left(1-\gamma^{5}\right)\left[\sum_{j=1}^{n}\left[\left(1-\frac{1}{2} \xi^{*} \xi^{T}\right) V_{1}\right]_{i j} \nu_{j}\right. \\
& \left.+\sum_{j=n+1}^{2 n}\left(\xi^{*}\right)_{i j} N_{j}\right]
\end{aligned}
$$

and

$$
\mathcal{L}_{n c}=-\frac{g}{4 \cos \theta_{W}} Z_{\mu} \sum_{i=1}^{n} \sum_{j, k=n+1}^{2 n} \bar{N}_{k} \gamma^{\mu}\left(1-\gamma^{5}\right)\left(\xi^{*} V_{2}\right)_{k i}^{\dagger}\left(\xi^{*} V_{2}\right)_{i j} N_{j}
$$

where we call $l_{i}$ the usual charged leptons, $\nu_{i}$ the light neutrinos and $N_{i}$ the heavy neutrinos.

In order to have a general situation we consider the mixing of an "electrontype light neutrino" and a new heavy Majorana neutrino with a mixing parameter fixed only by phenomenological consequences [8]. The most stringent 
bounds come from the LEP data on the $Z^{0}$ properties 9 . They are consistent with a bound of the order of $\sin ^{2} \theta_{\text {mix }}<10^{-2}-10^{-3}$.

Single heavy neutrino can be produced via:

$$
p+p \longrightarrow e^{-\bar{N}} X \longrightarrow e^{-} e^{-} W^{+} X
$$

We can also have same-sign electrons in pair production of heavy Majorana neutrinos

$$
p+p \longrightarrow N \bar{N} \longrightarrow e^{-} e^{-} W^{+} W^{+} Z
$$

but supressed by a term $\sin ^{4} \theta_{m i x}$. If the heavy neutrino comes from the muon family we will have a same-sign dimuon in the final state.

For reaction (8) one straightforward calculate [10] Drell-Yan cross sections. For Majorana neutrinos we have the charged current decay $N \longrightarrow l^{ \pm} W^{\mp}$. This gives a very clear final signature with two same sign charged leptons in the final state. The final signature for heavy neutrino decay with the highest branching ratio will be given by the hadronic channel $N \longrightarrow l^{ \pm} W^{\mp} \longrightarrow l^{ \pm}+$ hadrons. This is an interesting signature because there is no missing energy in the final state, and hadronic jet must have an invariant mass of $M_{W}^{2}$.

We have calculated cross sections and distributions using standard Monte Carlo methods. We show in figure 1 the total cross section at LHC energies for single heavy neutrino production. We employ the Gluck-Reya-Vogt parton distributions functions[11]. If we consider an annual luminosity of $100 \mathrm{fb}^{-1}$ and a rate of 10 events/year as feasible we see that LHC can detect Majorana neutrinos with mass up to $1.4 \mathrm{TeV}$. Of course this is tied to a mixing angle of $10^{-2}$. For the more stringent bound on the mixing angle $\sin ^{2} \theta_{\text {mix }}=10^{-3}$ we can reach masses up to $800 \mathrm{GeV}$. If no such neutrino is found this result can improve considerably the forbidden mass-angle region. There is an important effect on the total cross section due to the phase space for single heavy neutrino production. This is most clearly show in the variable $\tau=x_{1} x_{2}=\frac{\hat{s}}{s}$. The dominant region is at small $\tau$ and the kinematical threshold for single production at $M_{N}^{2}$ implies a larger cross section relative to pair production with threshold at $4 M_{N}^{2}$. This is shown in figure 2 . In figure 3 we show the primary lepton angular distribution. We call $\theta_{1}$ the angle of the outgoing lepton with the beam. For lighter neutrino mass it is peaked in the beam direction. As the heavy neutrino mass increases, this effect becomes smaller. The secondary lepton angular distribution follows the same pattern. In order 
to check if the cross section is still significative after an angular cut in the beam direction, we impose an angular cut for all the final particles (leptons and hadrons). The fraction of the signal outside this cone is shown in figure 4.

The most favored final signature will be $l^{ \pm} l^{ \pm}$hadrons, with the hadronic jet showing a high $P_{T}$ distribution peak around $\frac{M_{N}}{2}$ as shown in figure 5 .

In conclusion we have shown that the same-sign dileptons at the LHC energies can give a clear signal for heavy Majorana neutrinos with masses in the region of a few hundreds of $\mathrm{GeV}$ up to $1.4 \mathrm{TeV}$. The hadronic $P_{T}$ distribution gives a precise determination of the heavy neutrino mass.

\section{References}

[1] J.W.F.Valle, Nucl. Phys. (Proc. Suppl) 11(1989) 118.

[2] D.A.Dicus and P.Roy, Phys.Rev. D44(1991) 1593;

E.Ma and J.Pantaleone, Phys. Rev. D40(1989) 2172.

[3] M.Acciarri et al, L3 Collaboration, CERN-PPE/96-38;

D.Buskulik et al, ALEPH Collaboration, CERN-PPE/96-87;

G.Alexander et al, OPAL Collaboration, CERN-PPE/96-93.

[4] L.N.Chang,D.Ng and J.N.Ng, Phys. Rev. D50(1994) 4589;

C.Jarlskog, Phys. Lett. B241(1990) 579.

[5] W.Buchmüller and T.Yanagida,Phys. Lett. B302(1993) 240;

M.C.Gonzalez-Garcia,O.J.P.Éboli,F.Halzen and S.F.Novaes, Phys. Lett. B280(1992) 313.

[6] J.Schechter and J.W.F.Valle, Phys.Rev. D25 (1982) 774.

[7] D.Tommasini,G.Barenboim,J.Bernabéu and C.Jarslkog, Nuc. Phys. B444(1995) 451;

W.Buchmüller,C.Creub and P.Minkovski, Phys.Lett B267(1991) 355. 
[8] Y.A.Coutinho,J.A.Martins Simões and C.M.Porto, to appear in Z.Phys. C.

[9] E.Nardi,E.Roulet and D.Tommasini,Phys.Lett. B207(1994) 319;

P.Bamert,C.P.Burgess and I.Maksymyk, Phys.Lett. B356(1992) 282.

[10] A.Djouadi, Z. Phys. C63(1994) 317.

[11] M.Glück,E.Reya and A.Vogt, Z. Phys. C67(1995) 433. 
Acknowledments

This work was partially supported by CNPq, FINEP and FUJB.

\section{Figure Captions}

- 1. Total cross sections for pair and single heavy Majorana neutrinos at $\sqrt{s}=14 \mathrm{TeV}$ and $\sin ^{2} \theta_{\text {mix }}=10^{-2}$.

- 2.a Tau distribution for a single heavy Majorana neutrino.

- 2.b Tau distribution for a pair of heavy Majorana neutrinos.

- 3. Angular distribution of the primary lepton relative to the beam axis.

- 4. Total cross sections with an angular cut on the dileptons and hadronic jet.

- 5. Hadronic $P_{T}$ distributions for several heavy neutrino masses. 


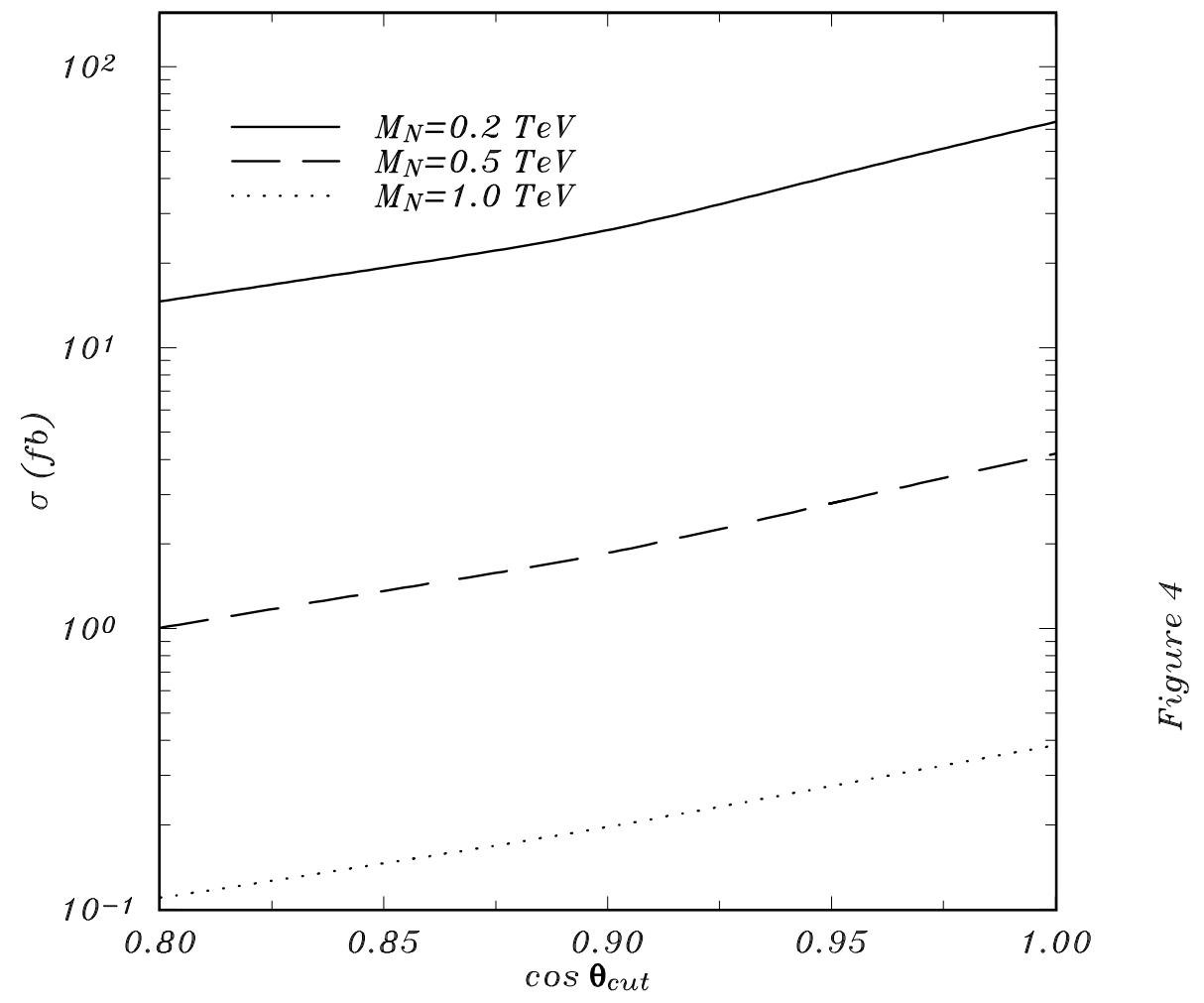




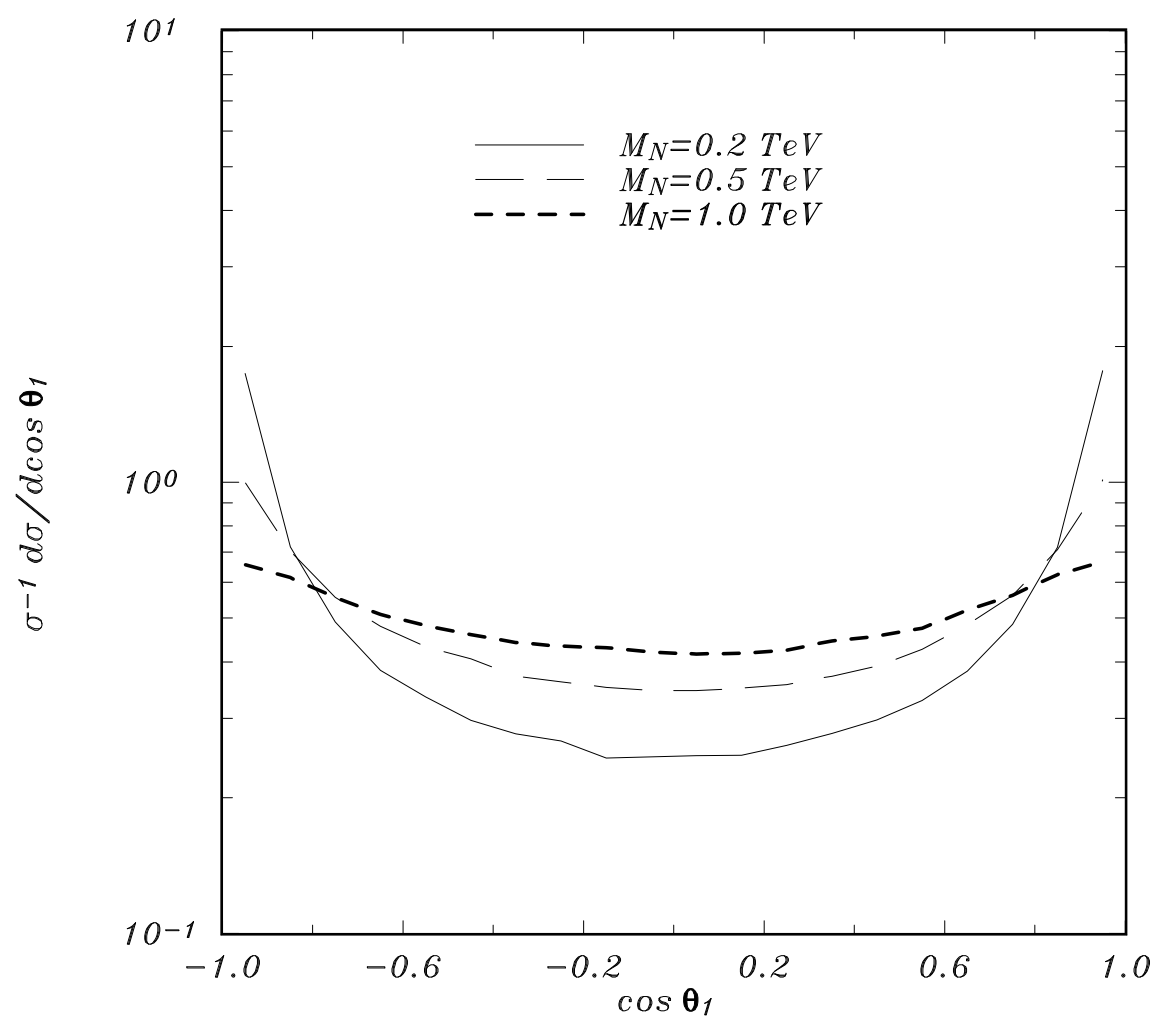




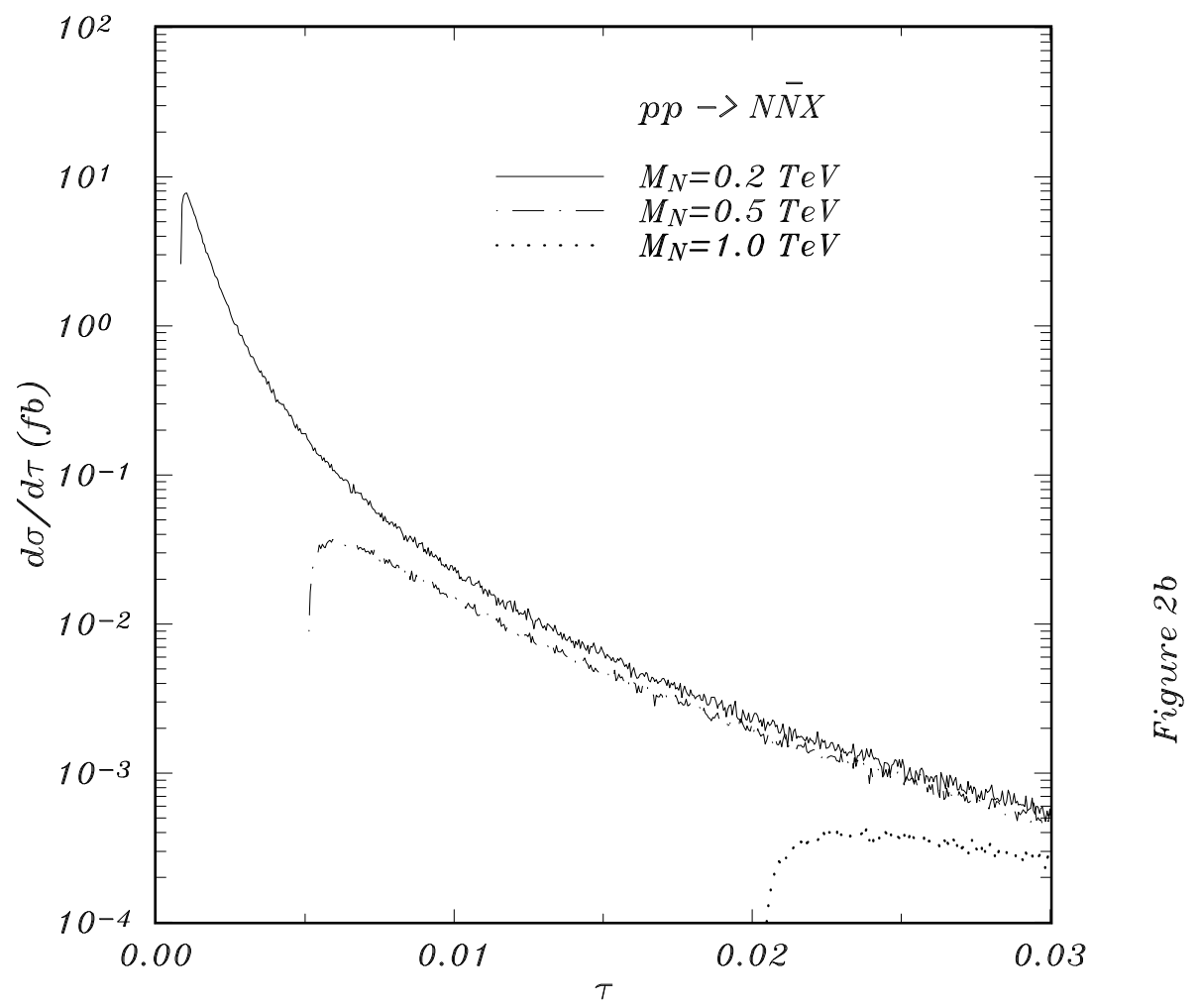




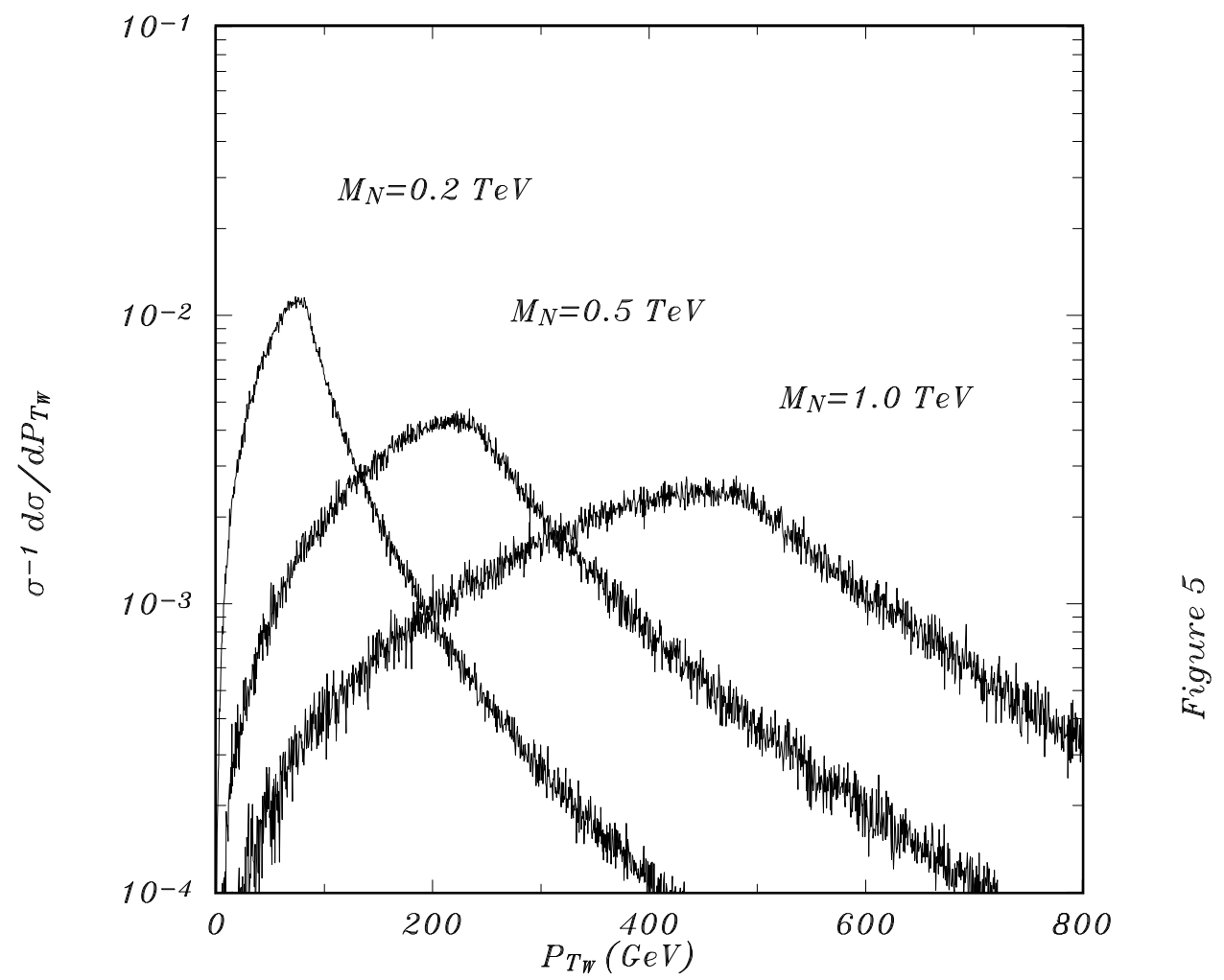




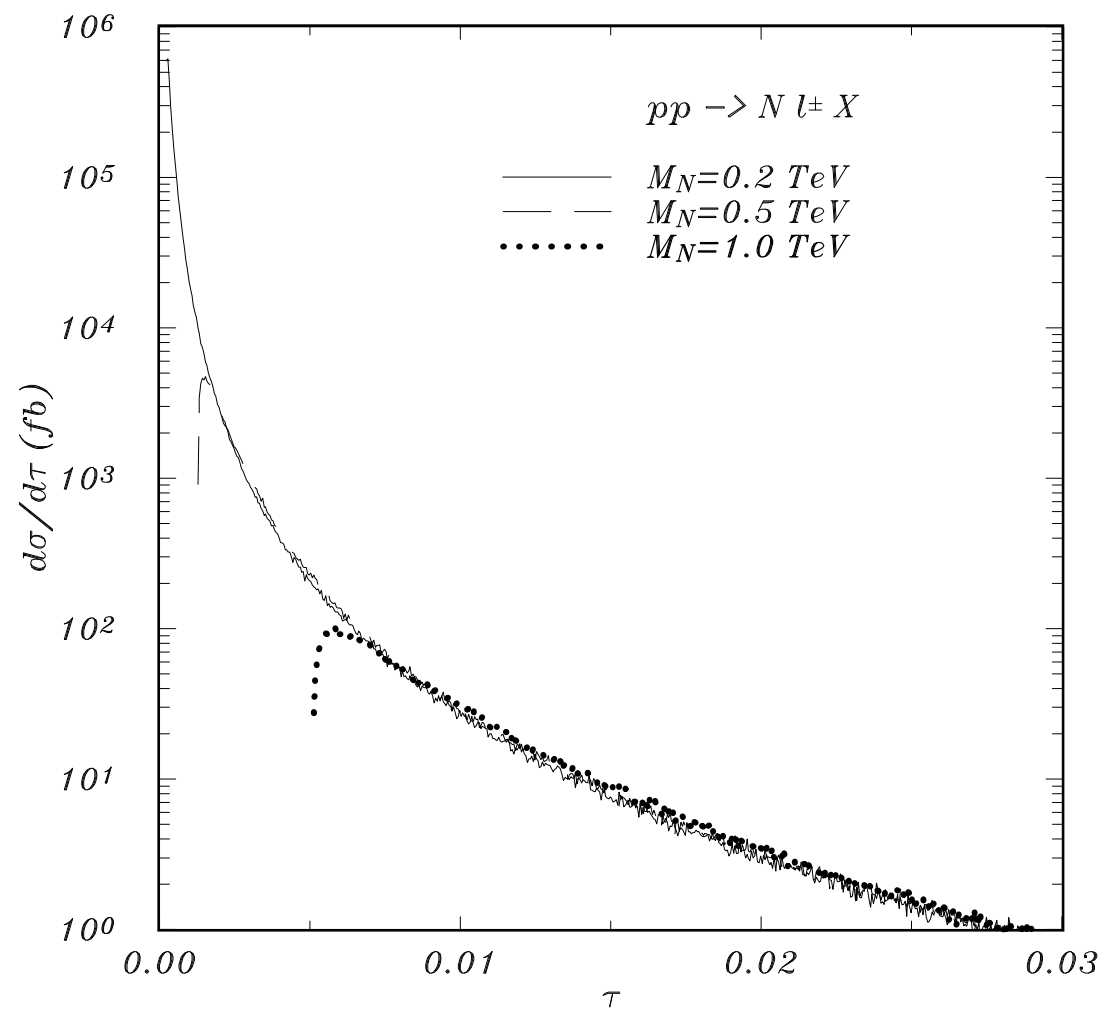




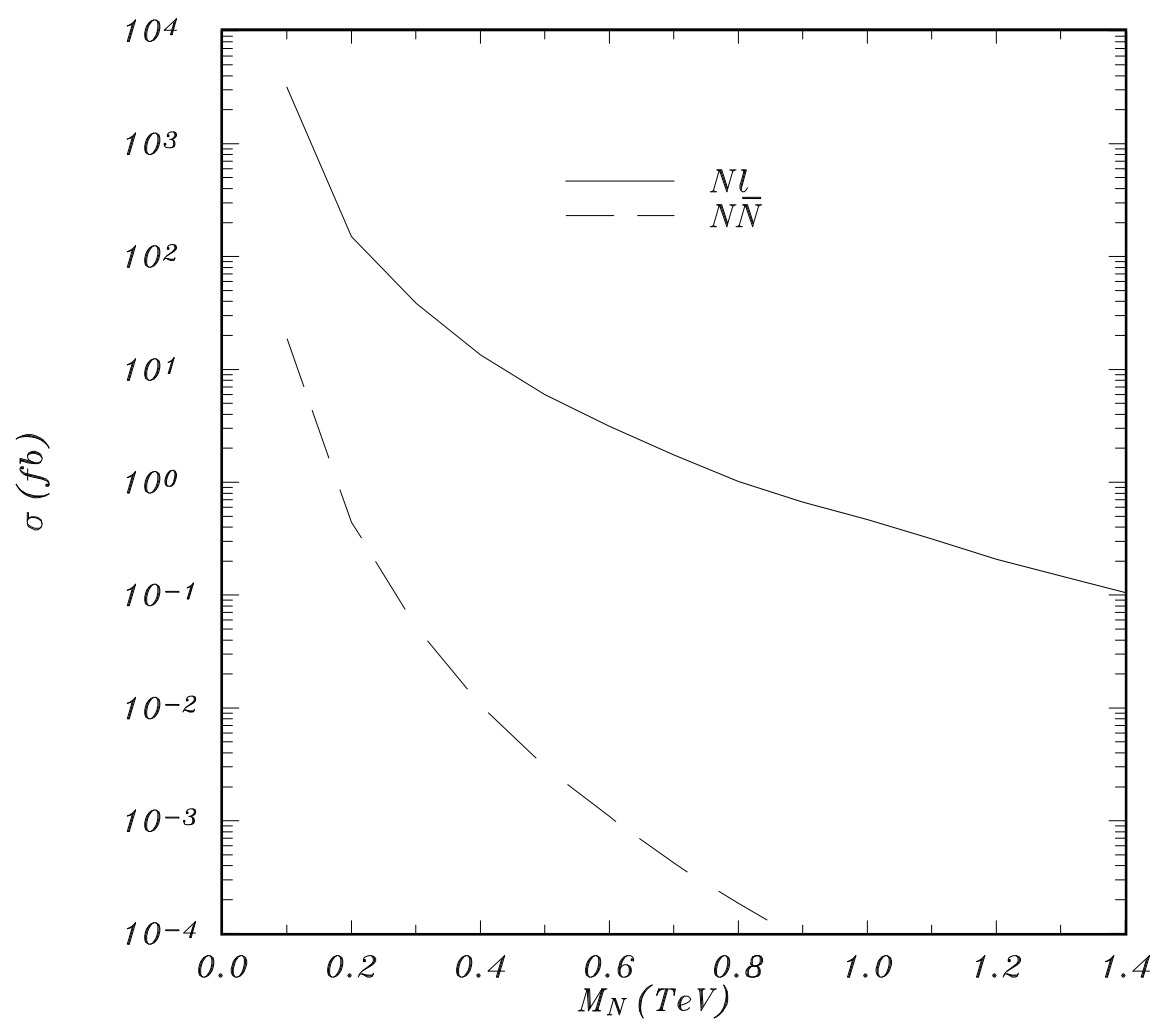

-
5
5
5
0
0 